\title{
Fadiga e qualidade de vida em bailarinos profissionais de dança de salão no Brasil
}

\author{
Fatigue and quality of life among professional ballroom dancers \\ in Brazil
}

\author{
Allana Alexandre Cardoso (https://orcid.org/0000-0002-0280-7567) ${ }^{1}$ \\ Nycolle Martins Reis (https://orcid.org/0000-0002-3925-9357) ${ }^{2}$ \\ Jaqueline da Silva (https://orcid.org/0000-0002-5248-3679) ${ }^{1}$ \\ Adriano Borgatto (https://orcid.org/0000-0001-6280-2525) ${ }^{3}$ \\ Alexandra Folle (https://orcid.org/0000-0001-8972-6075) ${ }^{2}$ \\ Rui Resende (https://orcid.org/0000-0003-4314-0743) ${ }^{1}$ \\ Adriana Coutinho de Azevedo Guimarães (https://orcid.org/0000-0001-5167-2921) ${ }^{2}$
}

${ }^{1}$ Programa de Pós-

Graduação em Educação

Física, Universidade Federal

de Santa Catarina (UFSC).

Campus Universitário

s/n, Trindade. $88040-900$

Florianópolis SC Brasil.

allana.alexandre@gmail.com

${ }^{2}$ Programa de Pós-

Graduação em Ciências

do Movimento Humano,

Centro de Ciências da Saúde

e do Esporte, Universidade

do Estado de Santa

Catarina. Florianópolis SC

Brasil.

${ }^{3}$ Departamento de

Informática e Estatística,

UFSC. Florianópolis SC

Brasil.

\begin{abstract}
The scope of this study was to analyze the relationship between quality of life, fatigue and injuries among professional Brazilian ballroom dancers. A total of 320 professionals from the five regions of Brazil participated, and the data were collected through a self-administered online questionnaire concerning general information, WHOQOL-bref, Yoshitake Fatigue Questionnaire and Injury Identification in Dancers Questionnaire. For data analysis, descriptive statistics were used, followed by inferential analysis and the Multiple Linear Regression test. The results show that greater fatigue is associated with a negative quality of life, revealing that, for each additional increment in total fatigue, the quality of life is expected to decrease by 0.218 points in the physical domain; 0.226 points in the psychological domain; 0.420 points in the social domain; and 0.304 points in the environmental domain. In contrast, the incidence and intensity of injuries were not associated with quality of life. The conclusion drawn is that the context of professional ballroom dance can influence both the presence of fatigue and the quality of life. It is suggested that health professionals working with these dancers perform multifactorial interventions in order to reduce fatigue, attenuating its effects on quality of life.
\end{abstract}

Key words Dancing, Fatigue, Injuries, Quality of life
Resumo Objetivou-se analisar a relação entre a qualidade de vida, a fadiga e as lesões em bailarinos brasileiros profissionais de dança de salão. Participaram 320 profissionais das cinco regiões do Brasil, sendo a coleta de dados realizada por meio de um questionário online autoaplicável acerca das informações gerais, WHOQOL-bref, Questionário de Fadiga de Yoshitake e Questionário de Identificação de Lesões em Bailarinos. Para análise dos dados utilizou-se a estatística descritiva, seguida da análise inferencial e do teste de Regressão Linear Múltipla. Os resultados demonstram que uma maior fadiga está associada a uma qualidade de vida negativa, evidenciando-se que, a cada valor a mais na fadiga total, espera-se que a qualidade de vida diminuisse 0.218 pontos no domínio físico; 0.226 pontos no domínio psicológico; 0.420 pontos no domínio social; e 0.304 pontos no dominio ambiental. Em contrapartida, a incidência e a intensidade de lesões não foram associadas com a qualidade de vida. Conclui-se que o contexto da dança de salão profissional pode influenciar tanto na presença de fadiga quanto na qualidade de vida. Sugere-se aos profissionais de saúde que atuam com estes bailarinos através de intervenções multifatoriais objetivando diminuir a fadiga, atenuando os efeitos desta na qualidade de vida.

Palavras-chave Dança, Fadiga, Lesões, Qualidade de vida 


\section{Introdução}

A dança de salão, caracterizada como uma atividade física sensoriomotora, envolve a integração de diferentes habilidades e resulta da exploração de possibilidades articulares que proporcionam variadas sensações físicas e psíquicas desencadeadas pelo movimento ${ }^{1}$, podendo ainda motivar e influenciar seus praticantes em diferentes aspectos, além de possibilitar o convívio e ampliar as relações sociais. É por meio de movimentações esteticamente belas, complexas e elaboradas que bailarinos profissionais projetam sua arte, expressando seus movimentos com elevados níveis de aptidão física e diferentes capacidades motoras.

No que se refere ao mercado de trabalho e às áreas de atuação, bailarinos, dançarinos, coreógrafos e professores integram a categoria de “artistas”, profissão regulamentada pela Lei no 6.533 , de 24 de maio de $1978^{2}$, e pelo Decreto no 82.385, de 05 de outubro de $1978^{3}$. Dessa forma, considera-se o profissional, perante os aspectos legais, aquele que detém um registro na Delegacia Regional do Trabalho (DRT), discriminado como artista nas distintas funções previstas, especificamente: bailarino ou dançarino; coreógrafo; assistente de coreógrafo; ensaiador e maître de balé. De acordo com a Classificação Brasileira de Ocupações $(\mathrm{CBO})^{4}$, a partir de 2002 estes profissionais passaram a ser denominados como "artistas da dança”. Todavia, indicadores importantes da profissão e organização da classe como condições trabalhistas, carga horária, piso salarial, dentre outras informações adjacentes ainda são desconhecidas no panorama nacional.

Sabe-se que, no âmbito profissional, a fim de atingir este alto padrão estético do movimento, a dança está associada à uma intensa rotina de treinamento, corroborando para que assim como atletas, bailarinos profissionais desenvolvam um elevado limiar de tolerância à dor ${ }^{5,6}$ e encontremse sujeitos a alta prevalência de lesões. Alguns estudos epidemiológicos relatam que até $63 \%$ dos estudantes de dança já sofreram alguma lesão ${ }^{7}$, sendo que a estimativa de prevalência para bailarinos profissionais pode variar de $40 \%$ a $84 \%^{8}$, fato este que pode ser explicado pelas exigências físicas a que são submetidos em sua carreira ${ }^{9}$.

Mceldowney et al. ${ }^{10}$ destacam ainda, ao avaliar bailarinos do Trinity Laban Conservatoire of Music and Dance, os efeitos da fadiga muscular no controle do equilíbrio como um dos principais contribuintes para ocorrência de lesões. Efeitos estes relacionados à exaustão física e emocional, decorrentes dos intensos períodos de treinamento e das exigências na perfeita execução de movimentos ${ }^{11}$, implicando inclusive em uma pior qualidade de vida para bailarinos fatigados $^{12,13}$.

Tendo isto posto, acredita-se que a performance artística somada à intensa rotina de treinamento possa trazer alguns prejuízos relacionados à qualidade de vida, ao estado de fadiga e às lesões de bailarinos profissionais de dança de salão. Assim, alerta-se para a necessidade de que estas temáticas, vinculadas ao contexto profissional da modalidade, sejam mais exploradas no meio científico, a fim de elucidar, conscientizar e contribuir para melhoria da saúde destes bailarinos. Desta forma, o presente estudo, considerado pioneiro em sua área, teve como objetivo analisar a relação entre a qualidade de vida, a fadiga e as lesões em bailarinos profissionais de dança de salão no Brasil.

\section{Materiais e métodos}

De delineamento transversal, o estudo teve sua aprovação no Comitê de Ética em Pesquisa envolvendo Seres Humanos (CEPSH) da Universidade do Estado de Santa Catarina. Devido a inexistência de órgãos que regulamentem a dança de salão profissional no Brasil, bem como de um controle das academias de dança de salão para busca destes profissionais, não foi possível obter o $\mathrm{N}$ da população para a realização do cálculo amostral. A amostra foi escolhida por conveniência a partir dos registros disponibilizados de Associações Regionalizadas em meio online, tais como Associação Nacional de Dança de Salão (ANDANÇAS); Comissão Brasileira da Dança de Salão (CBDS); Associação Catarinense de Dança de Salão (ACADS) e Associação dos Profissionais e Dançarinos de Salão do Rio de Janeiro (APDS), para realização do convite aos profissionais cadastrados. Em que pese existir, nos quadros da Classificação Brasileira de Ocupações pelo Ministério do Trabalho e Emprego $(\mathrm{CBO})^{4}$ a profissão bailarino, adotou-se para esta pesquisa o referido termo "bailarinos profissionais".

Desta forma, a amostra não probabilística intencional foi composta por 320 bailarinos profissionais de dança de salão, sendo 187 homens (58.4\%) e 133 mulheres (41,6\%), com média de idade de $31.48 \pm 8.63$ anos, das regiões Norte e Nordeste (20), Centro-Oeste (24), Sul (106) e Sudeste (170) do Brasil.

Determinou-se como critérios de inclusão: bailarinos profissionais; de ambos os sexos; 
maiores de 18 anos; praticantes da modalidade de dança de salão há no mínimo seis anos; que realizassem aulas, treinos e/ou ensaios de forma sistematizada numa frequência mínima de três vezes por semana; e que estivessem vinculados a uma companhia profissional de dança de salão e/ ou pertencessem ao corpo de professores de uma escola de dança. Como critérios de exclusão, estabeleceu-se: prática exclusiva de outras modalidades que não a dança de salão; e nível de escolaridade na classificação analfabeta.

\section{Coleta de dados}

Foram contactados 733 bailarinos profissionais, sendo que destes, 366 responderam o questionário completo e um total de 320 atenderam os critérios de inclusão.

O contato com as escolas, as companhias e os bailarinos profissionais autônomos estabeleceuse via telefone, e-mail e/ou redes sociais. Referente às escolas e às companhias, entrou-se em contato primeiramente com os diretores/coreógrafos e, se autorizado por estes, o questionário era enviado a cada bailarino identificado no grupo. Os bailarinos foram convidados a participar do estudo de forma voluntária e assim comunicados dos objetivos e dos procedimentos da investigação. Ao aceitarem participar, estes assinaram o Termo de Consentimento Livre e Esclarecido (TCLE) online. Neste aceite online, o bailarino dispunha de duas opções, especificamente "aceitar participar" ou "não aceitar participar”. Ademais, o bailarino poderia optar em receber ou não o retorno com os resultados da pesquisa, desde que disponibilizasse seus dados pessoais para contato posterior.

A coleta de dados ocorreu no período de novembro de 2016 a junho de 2017 por meio de um questionário autoaplicável online elaborado no GoogleDocs, no qual o link era encaminhado diretamente no e-mail de cada bailarino. Após o preenchimento e envio dos bailarinos, as respostas dos questionários eram enviadas para planilhas geradas pelo GoogleDocs e administradas pela pesquisadora principal da pesquisa.

\section{Instrumentos de pesquisa}

Para a coleta de dados, utilizou-se um questionário autoaplicável online, dividido em quatro blocos:

a) Informações gerais: idade, sexo, escolaridade, estado conjugal, ocupação funcional, nível econômico, tempo de contato e prática sistematizada da dança de salão, prática de outras dan- ças além da dança de salão e prática de atividade física. O Estrato Econômico dos participantes foi avaliado por meio do questionário Critério de Classificação Econômica Brasil (CCEB), proposto pela Associação Brasileira de Empresas de Pesquisa $(\mathrm{ABEP})^{14}$.

b) Qualidade de Vida: avaliada por meio do World Health Organization Quality of Life (WHOQOL-bref $)^{15}$, o qual consiste em 26 questões referentes às duas últimas semanas do indivíduo. Sendo destas, duas questões gerais relativas à qualidade de vida e as demais referentes aos domínios: físico (dor e desconforto, energia e fadiga, sono e repouso, mobilidade, atividades cotidianas, questões medicamentosas e capacidade no trabalho); psicológico (sentimentos positivos e negativos, questões cognitivas, autoestima e autoimagem e crenças pessoais); social (relações pessoais, suporte social e atividade sexual) e ambiental (segurança física, recursos financeiros, cuidados sociais e de saúde, acesso a informação e habilidades, recreação e lazer, ambiente do lar, condições do ambiente comum e transporte). A avaliação deu-se por uma escala de respostas do tipo Likert, na qual os valores são computados em escores que variam de 0 a $100 \%$, sendo que, quanto mais próximo de 100 , melhor a qualidade de vida do indivíduo.

c) Fadiga: avaliada pelo Questionário de Fadiga de Yoshitake, traduzido e utilizado no Brasil por Fischer et al. ${ }^{16}$. Correspondem ao questionário 30 questões de múltipla escolha divididas em três segmentos de avaliação, nomeadamente sonolência e falta de disposição no trabalho; dificuldade de concentração e atenção; e projeções da fadiga ao corpo. Cada segmento de avaliação possui 10 questões que variam de 10 pontos, referente a menor fadiga, até 50 pontos que caracteriza maior fadiga. $\mathrm{O}$ escore final geral varia de 30 pontos (menor fadiga) a 150 pontos (maior fadiga).

d) Lesões: avaliou-se por meio de um questionário auto administrado adaptado para bailarinos profissionais brasileiros por Lima et al. ${ }^{17}$. O Questionário de Identificação de Lesões em Bailarinos corresponde a 14 questões objetivas de múltipla escolha que abrangem a presença de lesões, a localização anatômica, a estrutura afetada, a frequência (uma, duas ou três lesões), a percepção dos fatores de risco, o tipo e/ou prática que ocasionou a lesão e as ações pós-lesão. Para a análise dos dados deste artigo foram consideradas apenas as respostas referentes à lesão 1 caracterizada como a primeira lesão citada pelo bailarino, ou seja, a mais relevante em sua carrei- 
ra profissional - e as questões relativas à incidência e à intensidade da lesão.

\section{Análise dos dados}

A análise estatística foi realizada por meio do pacote SPSS - IBM versão 20.0. Realizou-se primeiramente uma análise descritiva para conhecimento da distribuição dos dados em cada variável e posteriormente uma análise inferencial. Para associação da qualidade de vida com a fadiga e as lesões, utilizou-se o teste de Regressão Linear Múltipla, aplicando o método Enter de seleção de variáveis. O nível de significância adotado foi de $5 \%$.

\section{Resultados}

Ao traçar um perfil geral dos bailarinos profissionais de dança de salão do Brasil, percebeu-se que a maioria era do sexo masculino $(58,4 \%)$, com companheiros $(52,2 \%)$, pertencentes à classe média $(74,7 \%)$, haviam cursado o Ensino Superior completo $(53,1 \%)$, sendo que destes, $16,9 \%$ possuíam curso de pós-graduação (dados não apresentados). Estes possuíam entre seis e 10 anos de contato com a modalidade investigada $(50,6 \%)$ e mantinham a periodicidade da prática de cinco à sete vezes por semana $(53,8 \%)$. Além disso, $48,8 \%$ praticavam outra modalidade de dança, além da dança de salão, e 72,8\% desempenhavam outra atividade física, além da dança, sendo o exercício resistido a prática citada com mais adeptos $(49,8 \%)$ (dados não apresentados).

No que se refere aos domínios social $(70,39 \pm 19,95)$ e psicológico $(68,22 \pm 12,15)$, estes foram os que apresentaram os melhores escores dentro da qualidade de vida, seguidos da qualidade de vida total $(64,89 \pm 11,72)$ e dos domínios ambiental $(63,09 \pm 16,04)$ e físico $(57,85 \pm 11,63)$. Relativamente à ocorrência de lesões na amostra investigada, $64,1 \%$ relataram ter sofrido alguma lesão em decorrência da sua carreira profissional na dança de salão, em que destes, $41,3 \%$ citaram ter sofrido apenas uma lesão, $17,8 \%$ até duas lesões e $8,1 \%$ até 3 lesões (dados não apresentados).

Na Tabela 1, observa-se a associação entre os domínios da qualidade de vida com a fadiga e as variáveis incidência e intensidade da lesão. Verificou-se que, a cada valor a mais na fadiga total, espera-se que a qualidade de vida diminua 0,218 pontos no domínio físico $\left(\mathrm{R}^{2}=0,130 ; \mathrm{p}<0,001\right)$; 0,226 pontos no domínio psicológico $\left(R^{2}=0,120\right.$;

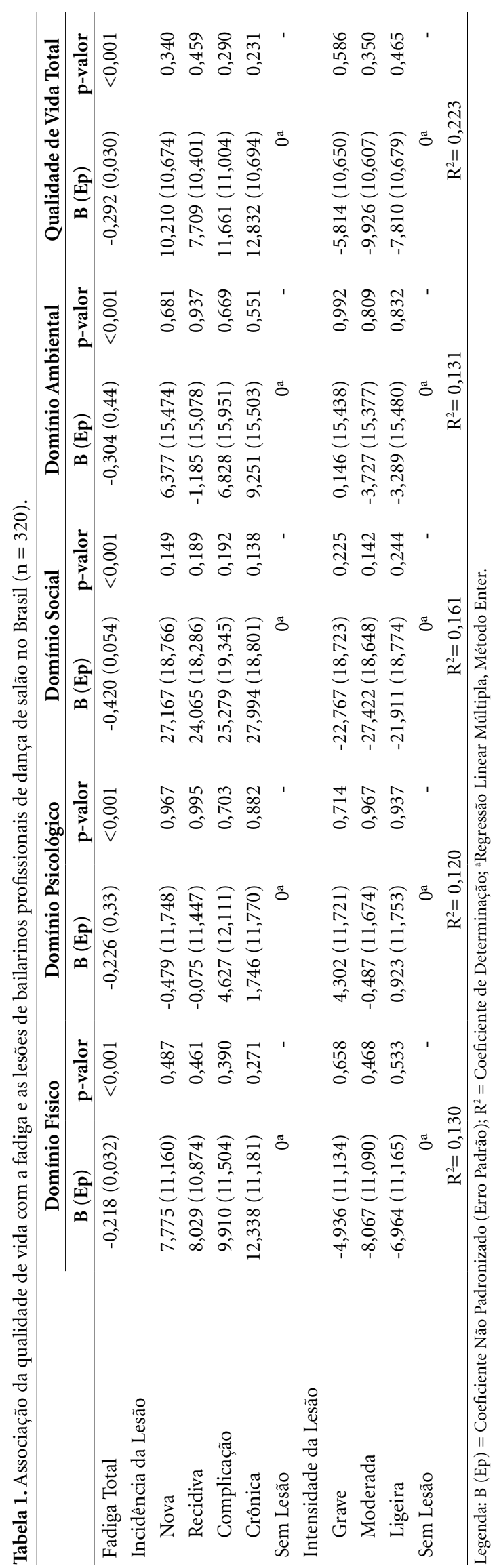


$\mathrm{p}<0,001) ; 0,420$ pontos no domínio social $\left(\mathrm{R}^{2}=0,161 ; \mathrm{p}<0,001\right) ; 0,304$ pontos no domínio ambiental $\left(\mathrm{R}^{2}=0,131 ; \mathrm{p}<0,001\right)$ e 0,292 pontos na qualidade de vida total $\left(\mathrm{R}^{2}=0,223 ; \mathrm{p}<0,001\right)$. Não foram encontradas associações quanto aos domínios da qualidade de vida e a incidência e a intensidade da lesão.

\section{Discussão}

Ao objetivar analisar a relação entre a qualidade de vida, a fadiga e as lesões em bailarinos profissionais de dança de salão no Brasil, evidenciouse que uma maior fadiga está associada a uma qualidade de vida negativa. Em contrapartida, a incidência e a intensidade de lesões não foram associadas com a qualidade de vida.

Sabe-se que a dança de salão, por meio da sua ideologia, desenvolve aspectos físicos, cognitivos e sociais ${ }^{18}$. Todavia, apesar de ser uma modalidade que potencializa os benefícios advindos de um estilo de vida ativo e saudável, salienta-se que a rotina de um bailarino profissional é demasiada intensa, exigindo muitas vezes, capacidade de interpretação e execução de movimentos complexos em meio a dores e desconfortos físicos ${ }^{5,6,8}$. Adicionalmente ao desgaste físico, ressalta-se a acentuada pressão para atingir os padrões plásticos esperados nos palcos e nos salões ${ }^{19}$. Fatores estes que podem levar bailarinos a um quadro de estresse psicofisiológico, incidindo diretamente na presença da fadiga e no aumento da susceptibilidade a lesões ${ }^{10,20}$.

Investigada recentemente por prejudicar o rendimento físico e mental de diferentes tipos de populações como trabalhadores ${ }^{21}$, atletas ${ }^{22}$, bailarinos profissionais das modalidades de ballet clássico ${ }^{23}$ e contemporâneo ${ }^{24}$, a fadiga relaciona-se também aos prejuízos na qualidade de $v$ ida $^{25}$. Favorecendo estes achados, os resultados do presente estudo evidenciaram que uma maior presença de fadiga se associou a uma pior qualidade de vida. Isto, pois, a sensação generalizada de cansaço, dor e estresse, bem como a falta de motivação para o trabalho físico e/ou mental ${ }^{26}$, pode colaborar para que bailarinos fatigados se sintam indispostos e menos motivados à prática, prejudicando desta maneira, os aspectos de sua qualidade de vida.

Comumente vinculada aos esportes de resistência e de alto desempenho, a fadiga muscular é tipicamente quantificada pela diminuição da força máxima ou da falta de percepção de energia muscular na manutenção da tarefa ${ }^{27}$. Apesar de neste estudo não terem sido utilizadas medidas diretas para a avaliação da fadiga, os resultados apontam uma relação entre maior presença de fadiga com piores escores no domínio físico da qualidade de vida dos bailarinos. Fato este que pode ser explicado pela especificidade da dança, que combina atividades de alta intensidade de forma intermitente, utilizando metabolismo aerobio e anaeróbio e deixando estes profissionais suscetíveis à fadiga ${ }^{28}$. Notabiliza-se também que bailarinos fatigados podem sentir desconfortos oriundos do domínio físico da qualidade de vida, visto que este engloba questões relacionadas à falta de energia, às dores no corpo, ao sono, ao repouso, às questões medicamentosas, à capacidade de desempenhar as tarefas, entre outras, o que elucida a associação deste domínio com a fadiga.

Ainda, no que concerne a intensa demanda de exercícios físicos, principalmente quando se aproximam de shows, espetáculos ${ }^{29}$ e competições ${ }^{30}$, a fadiga pode relacionar-se aos prejuízos na performance, além de estar associada a uma série de fatores que influenciam os aspectos psicológicos destes bailarinos. No mesmo sentido, o presente estudo notou a associação da fadiga com o domínio psicológico da qualidade de vida. Uma hipótese para este achado seria o esgotamento psicológico e emocional enfrentado por estes profissionais, uma vez que estes lidam diariamente com níveis elevados de ansiedade e estresse, decorrentes do medo de errar ou esquecer a sequência coreográfica, exposição ao público ou a jurados, sendo constantemente submetidos à observações, a julgamentos e à opiniões, o que pode vir a influenciar negativamente o desempenho psicológico ${ }^{30}$, além da falta de repouso necessário para as atividades cognitivas e mentais desempenhadas no dia a dia.

Ademais neste estudo, quando na presença de fadiga, bailarinos profissionais de dança de salão também apresentaram piores escores no domínio social da qualidade de vida. Supõe-se que, as exigências sociais associadas à extrema cobrança por rendimento físico, artístico, bem como pela presença nos ensaios, viagens e apresentações, podem influenciar os relacionamentos pessoais destes profissionais com seus familiares, cônjuges, filhos e/ou amigos. Uma vez que, bailarinos tendem a se sentir afastados de seus vínculos sociais devido ao cansaço físico e mental, as exigências e as demandas do âmbito profissional da dança.

Ainda neste estudo, bailarinos fatigados apresentaram piores escores no domínio ambiental da qualidade de vida, no qual o ambiente da 
dança, as apresentações e as competições, com as intensas iluminações e o som alto podem gerar no bailarino situações de ansiedade e estresse ${ }^{30}$. Conjectura-se também que a intensa convivência com seu parceiro/demais integrantes do grupo durante os recorrentes ensaios e viagens para apresentações, eventos, cursos e afins ao qual são submetidos, torne o ambiente propício para a ansiedade e o estresse, influenciando assim este domínio da qualidade de vida.

Para além disso, a fadiga também é tida como um dos principais fatores que levam a desencadear lesões em bailarinos ${ }^{31,32}$. Todavia, apesar de $64,1 \%$ dos bailarinos de dança de salão terem sofrido alguma lesão decorrente da sua carreira profissional, fazendo desta uma variável importante neste estudo, não foram encontrados dados significativos para a associação da intensidade e da incidência da lesão com a qualidade de vida e seus domínios. Corroborando ao alto índice na presença de lesões da amostra investigada no presente estudo, Pellicciari et al..$^{33}$ investigaram a presença de lesões em bailarinos da mesma modalidade no contexto internacional, revelando que $47,7 \%$ dos bailarinos reportaram até uma lesão decorrentes da prática.

Entretanto, apesar da alta demanda de atividades e fatores estressantes vinculados a prática profissional da dança, ao analisar a qualidade de vida de forma isolada, verificou-se que, de forma geral, bailarinos profissionais de dança de salão possuem uma boa qualidade vida, com destaque para os domínios social e psicológico. Dados estes que vão ao encontro dos achados de Machado et al. ${ }^{34}$, os quais ao avaliarem a qualidade de vida de 402 praticantes não profissionais de dança de salão em contexto nacional, encontraram igual destaque para estes domínios.

Por ser dançada em pares, acredita-se que a dança de salão proporciona melhorias tanto para praticantes no âmbito profissional quanto no lazer. Justamente pela questão da busca por avanços intra e interpessoais serem tidos como um dos fatores de aderência à prática desta modalidade ${ }^{18}$. Uma hipótese para os escores mais elevados nos domínios social e psicológico refere-se ao fato da dança a dois não se limitar somente às aulas regulares, aos estudos e aos ensaios sistematizados, bailarinos profissionais e amadores encontramse em festas e bailes, tornando sua prática efetiva na construção das relações sociais, com possíveis benefícios no âmbito social e psicológico da qualidade de vida. Lakes et al. ${ }^{1}$ ratificam que dançar com frequência e por longos períodos está associado ainda a melhorias na aptidão física, no humor, na autoconfiança, e no funcionamento social e cognitivo.

Embora seja evidente que a dança de salão proporcione experiências que ampliam o potencial estético, artístico e cultural de seus adeptos ${ }^{34}$, no contexto profissional, ainda se faz necessário a adoção de hábitos que favoreçam a saúde desses bailarinos, tais como: o uso correto da indumentária - principalmente calçados adequados -, repouso pré e pós ensaios/aulas/espetáculos e estimulação de ambientes tranquilos para equilibrar os níveis de estresse e ansiedade. Adicionalmente, Wainwright et al. ${ }^{35}$ alertam que é de fundamental importância o adequado dimensionamento entre força e flexibilidade, combinado com a aptidão de habilidades técnicas na execução dos movimentos e prática de atividades compensatórias ${ }^{5}$, a fim de evitar que as lesões musculoesqueléticas ocorram neste meio.

Desta forma, indica-se que programas específicos sejam desenvolvidos para essa população, objetivando diminuir a presença de fadiga e o desencadeamento de lesões, ressaltando-se a necessidade de que estes bailarinos tenham acesso a ambientes seguros para a prática, para além de serem incentivados a aquecer-se e alongar-se durante suas aulas/ensaios, além de receberem um tratamento imediato e de qualidade quando na presença de lesões ${ }^{32}$.

Neste estudo evidenciou-se como limitação a utilização de medidas indiretas para avaliação dos bailarinos, por tratar-se de uma pesquisa online. Contudo, ressalta-se que a opção de uma coleta de dados online deu-se pela pesquisa caracterizar-se de abrangência nacional, objetivando acessar o maior número de bailarinos profissionais e não contar com nenhum tipo de apoio financeiro. Pontos positivos deste estudo também devem ser identificados, especialmente na limitação de estudos na literatura, em que apesar das evidências enfatizarem os benefícios da dança na melhoria e na manutenção de uma qualidade de vida positiva nos mais diversos aspectos e populações ${ }^{34,36,37}$, poucas são as investigações que se dedicam a investigar a dança de salão no seu contexto profissional ${ }^{1}$. Fato que foi estabelecido pelo presente estudo.

\section{Considerações finais}

Bailarinos com maior presença de fadiga apresentam piores escores nos domínios psicológico, físico, social e ambiental da qualidade de vida, indicando assim que o contexto da dança de salão 
profissional pode influenciar tanto na presença de fadiga quanto nos domínios da qualidade de vida. Não foram encontradas associações entre a incidência e a intensidade da lesão com a qualidade de vida dos bailarinos.

Faz-se importante salientar que os resultados obtidos, pelo estudo em questão, objetivam também auxiliar na conscientização e no conhecimento dos bailarinos, coreógrafos, diretores e demais envolvidos nas companhias e escolas de dança, no que concerne à importância na manutenção de hábitos saudáveis para a minimização de fatores prejudiciais ao estado de saúde geral destes profissionais. Nesse sentido, sugere-se aos profissionais de saúde que atuam com estes bailarinos a realização de intervenções psicológicas e físicas, a fim de diminuir a presença da fadiga, bem como atenuar os efeitos desta variável na qualidade de vida destes bailarinos, favorecendo a estes uma carreira saudável e segura.

\section{Colaboradores}

AA Cardoso: concepção, delineamento, análise crítica, interpretação dos dados, redação do artigo, revisão e aprovação da versão submetida. NM Reis: análise, interpretação dos dados, redação do artigo e aprovação da versão submetida. J Silva: revisão, redação do artigo e aprovação da versão submetida. A Borgatto: delineamento, análise crítica, interpretação dos dados, redação do artigo e aprovação da versão submetida. A Folle: análise crítica, redação do artigo, revisão e aprovação da versão submetida. R Resende: análise crítica, redação do artigo, revisão e aprovação da versão submetida. ACA Guimarães: delineamento, análise crítica, interpretação dos dados, redação do artigo e aprovação da versão submetida.

\section{Financiamento}

Salienta-se ainda que o presente trabalho foi realizado com apoio da Coordenação de Aperfeiçoamento de Pessoal de Nível Superior- Brasil (CAPES) - Código de Financiamento 001.

\section{Referências}

1. Lakes KD, Marvin S, Rowley J, Nicolas MS, Arastoo S, Viray L, Orozco A, Jurnak F. Dancer perceptions of the cognitive, social, emotional, and physical benefits of modern styles of partnered dancing. Complement Ther Med 2016; 26:117-122.

2. Brasil. Lei no 6.533, de 24 de maio de 1978. Dispõe sobre a regulamentação das profissões de Artistas e de técnico em Espetáculos de Diversões, e dá outras providências. Diário Oficial da União 1978; 25 maio.

3. Brasil. Decreto no 82.385 , de 5 de outubro de 1978 . Regulamenta a Lei no 6.533, de 24 de maio de 1978 , que dispõe sobre as profissões de Artista e de Técnico em Espetáculos de Diversões, e dá outras providências. Diário Oficial da União 1978; 6 out.

4. Brasil. Ministério do Trabalho. Classificação Brasileira de Ocupações (CBO) [Internet]. 2002 [acessado 2019 Mar 26]. Disponível em: http://www.mtecbo.gov.br/

5. Dore BF, Guerra RO. Sintomatologia dolorosa e fatores associados em bailarinos profissionais. Rev Bras Med Esporte 2007; 13(2):77-80.

6. Couto AGA, Pedroni CR. Relação entre postura, queixa dolorosa e lesão em bailarinas clássicas. Terapia Manual 2013; 11(52):228-233.

7. Fernandes TFR, Lopez AD, Lopes AD. Lesões musculoesqueléticas no ballet: revisão sistemática. Terapia Manual 2011; 9(43):304-310.

8. Hincapie CA, Morton EJ, Cassidy JD. Musculoskeletal injuries and pain in dancers: a systematic review. Arch Phys Med Rehabil 2008; 89(9):1819-1829. 
9. Cardoso, AA, Reis NM, Marinho APR, Vieira MDCS, Boing L, Guimarães ACDA. Injuries in professional dancers: a systematic review. Rev Bras de Med Esporte 2017; 23(6):504-509.

10. McEldowney KM, Hopper LS, Etlin-Stein H, Redding E. Fatigue effects on quadriceps and hamstrings activation in dancers performing drop landings. J Dance Med Sci 2013; 17(3):109-114.

11. Costa VT, Pires DA, Ferreira Filho E, Noce F. Análise da síndrome de Burnout em bailarinos. Rev Educ Fis UEM 2014; 25(2):163-171.

12. Armstrong R, Brogden C, Milner D, Norris D, Greig M. Effect of Fatigue on Functional Movement Screening Performance in Dancers. Med Probl Perform Art 2018; 33(3):213-219.

13. Kenny SJ, Palacios-Derflingher L, Whittaker JL, Emery CA. The influence of injury definition on injury burden in preprofessional ballet and contemporary dancers. J Orthop Sports Phys Ther 2018; 48(3):185193.

14. Associação Brasileira de Empresas de Pesquisa (ABEP). Critério de classificação econômica Brasil [Internet]. [acessado 2019 Maio 22]. Disponível em: http://www.abep.org/novo/Content.aspx?ContentID $=835$

15. Fleck MPA, Louzada S, Xavier M, Chachamovich E, Vieira G, Santos L, Pinzon V. Aplicação da versão em português do instrumento abreviado de avaliação da qualidade de vida "WHOQOL-bref". Rev Saúde Pública 2000; 34(2):178-183.

16. Fischer FM, Borges NS, Rotenberg L, Latorre MRDO, Soares NS, Rosa PLFS, Nagai R, Landsbergis P. A (in) capacidade para o trabalho em trabalhadores de enfermagem. Rev Bras Med Trab 2005; 3(2):97-103.

17. Lima KA, Silva PHB, Barreto RR. Características das lesões em bailarinos e sua relação com a qualidade de vida. Movimenta 2014; 7(1):645-659.

18. Farencena EZP, Montelo Júnior D, Gross DGL, Rodrigues ESF. Dança de salão e sua contribuição para melhoria da saúde e qualidade de vida. Amazônia Sci Health 2016; 4(1):10-16.

19. Berndt C, Strahler J, Kirschbaum C, Rohleder N. Lower stress system activity and higher peripheral inflammation in competitive ballroom dancers. Biol Psychol 2012; 91(3):357-364.

20. Silva AMB, Enumo SRF. Dor e lesões em bailarinos adolescentes: revisão sistemática. Rev Dor 2016; 17(2):132-135.

21. Moore R, Gupta P, Duval N. Occupational fatigue: impact on anesthesiologist's health and the safety of surgical patients: as anesthesiologists we are frequently working in a stressful environment. Do you disagree with this? Rev Bras Anestesiol 2013; 63(2):167-169.

22. Wewelhove $\mathrm{T}$, Raeder C, Meyer T, Kellmann M, Pfeiffer M, Ferrauti A. Markers for routine assessment of fatigue and recovery in male and female team sport athletes during high-intensity interval training. PloS One 2015;10(10):e0139801.

23. Lima CD, Brown LE, Wong MA, Leyva WD, Pinto RS, Cadore EL, Ruas CV. Acute Effects of Static vs. Ballistic Stretching on Strength and Muscular Fatigue Between Ballet Dancers and Resistance Trained Women. J Strength Cond Res 2016; 30(11):3220-3227.
24. Liederbach M, Dilgen FE, Rose DJ. Incidence of anterior cruciate ligament injuries among elite ballet and modern dancers a 5 -year prospective study. Am J Sports Med 2008; 36(9):1779-1788.

25. Mota DDCF, Cruz DALM, Pimenta CAM. Fadiga: uma análise do conceito. Acta Paul Enferm 2005; 8(3):285-293.

26. Enoka RM, Duchateau J. Translating fatigue to human performance. Med Sci Sports Exerc 2016; 48(11):22282238.

27. Rutherford G, Manning P, Newton JL. Understanding Muscle Dysfunction in Chronic Fatigue Syndrome. $J$ Aging Res 2016; 2497348.

28. Rodrigues-Krause J, Krause M, Reischak-Oliveira Á. Cardiorespiratory Considerations in Dance: From Classes to Performances. J Dance Med Sci 2015; 19(3):91-102.

29. Liiv H, Jürimäe T, Mäestu J, Purge P, Hannus A, Jürimäe J. Physiological characteristics of elite dancers of different dance styles. Eur J Sport Sci 2014; 14(Supl. 1):S429-S436.

30. Pereira CSRF, Simas JPN, Boing L, Machado Z, Matias TS, Guimarães ACA. Nível de ansiedade em bailarinos pré e pós competição. $R B C M$ 2014; 22(4):116-125.

31. Rodrigues-Krause J, Cunha GS, Alberton CL, Follmer B, Krause M, Oliveira AR. Oxygen consumption and heart rate responses to isolated ballet exercise sets. $J$ Dance Med Sci 2014; 18(3):99-105.

32. McCabe TR; Hopkins JT; Vehrs P; Draper DO. Contributions of Muscle Fatigue to a Neuromuscular Neck Injury in Female Ballroom Dancers. Med Probl Perform Art 2013; 28(2):84-90.

33. Pellicciari L, Piscitelli D, De Vita M, D'Ingianna L, Bacciu S, Perno G, Lunetta L, Rosulescu E, Cerri CG, Foti C. Injuries Among Italian DanceSport Athletes: A Questionnaire Survey. Med Probl Perform Art 2016; 31(1):13-17.

34. Machado Z, Santos GR, Guimarães ACA, Fernandes S, Soares A. Qualidade de vida dos praticantes de dança de salão. Rev Bras Ativ Fís Saúde 2012; 17(1):39-45.

35. Wainwright SP, Williams C, Turner BS. Fractured identities: injury and the balletic body. Health (London) 2005; 9(1):49-66.

36. Carvalho T, Gonzáles AI, Sties SW, Carvalho GMD. Reabilitação cardiovascular, dança de salão e disfunção sexual. Arq Bras Cardiol 2013; 101(6):e107-e108.

37. Cepeda CC, Lodovico A, Fowler N, Rodacki AL. Effect of an Eight-Week Ballroom Dancing Program on Muscle Architecture in Older Adults Females. J Aging Phys Act 2015; 23(4):607-612.

Artigo apresentado em 29/01/2019

Aprovado em 02/05/2019

Versão final apresentada em 04/05/2019

Editores chefes: Romeu Gomes, Antônio Augusto Moura da Silva 